\title{
An Overview of Traffic Signs Recognition Methods
}

\author{
Prachi Dewan \\ Research Scholar \\ EECE Dept. \\ The North Cap \\ University \\ Gurugram, Haryana
}

\author{
Rekha Vig, PhD \\ EECE Dept. \\ The North Cap \\ University \\ Gurugram, Haryana
}

\author{
Neeraj Shukla, \\ $\mathrm{PhD}$ \\ EECE Dept. \\ The North Cap \\ University \\ Gurugram, Haryana
}

\author{
B. K. Das \\ Professor \\ EECE Dept. \\ The North Cap \\ University \\ Gurugram, Haryana
}

\begin{abstract}
Image processing has many areas of applications like in metrological prediction of weather conditions, medical science, artificial intelligence, robotics etc. Traffic signs recognition system is in fact the most happening area of research these days. As the world is moving towards the driverless vehicles, a more automated world is the utmost requirement for improving the road safety. Such systems can help the drivers on signs that they may not have noticed beforehand. These systems are so designed so that they consume less power and hence can be efficiently implemented on hardware. FPGA's are preferred over CPU and GPU due to its low cost and power, prototyping applications and next levels to ASIC's development. In this paper we have quoted some basic challenges in traffic signs recognition methods and summarized the various detection and recognition techniques for traffic signs. This paper divided the various methods into three categories: color-based, shape-based and learning- based. We have concluded that the Xilinx System Generator is the best tool while implementing on FPGA's. It is the fastest resource estimation tool in order to take full advantage of FPGA's resources. Finally, the hardware perspective of traffic signs implementation is briefly examined.
\end{abstract}

\section{General Terms}

Image pre-processing, Traffic Signs, Shape Extraction, Algorithm, Driver Assistance system

\section{Keywords}

Traffic Sign Recognition method, Field programmable gate array (FPGA), Xilinx system generator (XSG), Advanced Driver assistance system (ADAS), Machine learning techniques.

\section{INTRODUCTION}

Advanced Driver assistance system is a smart system designed to improve the safety and comfort of drivers. Every year thousands of traffic accidents occur around the world that causes loss of lives. When driving on a congested road, it is sometimes difficult to keep a track of the oncoming traffic and also what is behind us, while trying to maintain speed. Hence a system should be designed to detect all the traffic signs beforehand for display console in front of the driver. The increase in computing power of machines has made the real time detection and recognition of traffic signs possible.

Recognition of speed limit sign can inform the driver about the present speed limit and it can also alert the driver if a car is being driven faster than the speed limit. This appears to be easy as drivers can easily recognize all the signs because the color and shapes of the signs are very different from the natural environment. They are designed with specific colors and shapes, with a text or symbol in a high contrast to background. But the reality during sign detection by machines is different due to many limitations regarding shape, size and orientation of road signs. Sometimes they are partially hidden and this can lead to false detection The colors that are mostly used are blue and red for all warning and prohibitory signs. Moreover, the shapes of the signs are triangle or circle which are uncommon in the nature. These two unique features of traffic signs give motivation to design efficient algorithms for detection and recognition. In this paper, we have tried to compare all the technologies and tools used in designing a robust traffic signs detection system. Both the image processing and machine learning algorithms have to be improved to implement the real time constraints of traffic signs recognition system. Figure 1 shows the various prohibitory and warning road signs.

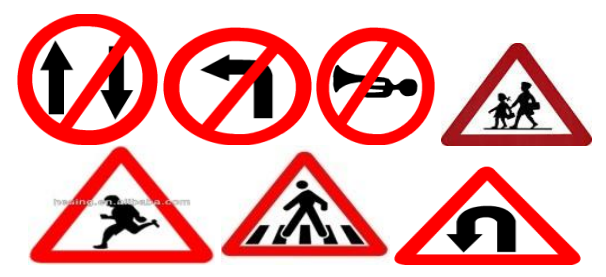

Figure 1:Various Warning and Prohibitory Signs

However, there are some problems while processing the images of signs. Some of the main problems are low camera resolution, imperfect sign state, illumination, and occlusion, weather conditions such as rain, snow or fog. All of these can affect the color analysis and shape extraction of signs. In order to correctly recognize the signs, the above mentioned challenges should be properly addressed. In most of the published papers, the system is designed using a three stage sequential approach i) preprocessing stage and then detection stage ii) recognition stage and iii) finally transmission( from one vehicle to another) stage. The various stages of traffic signs recognition system is shown in figure 2 .

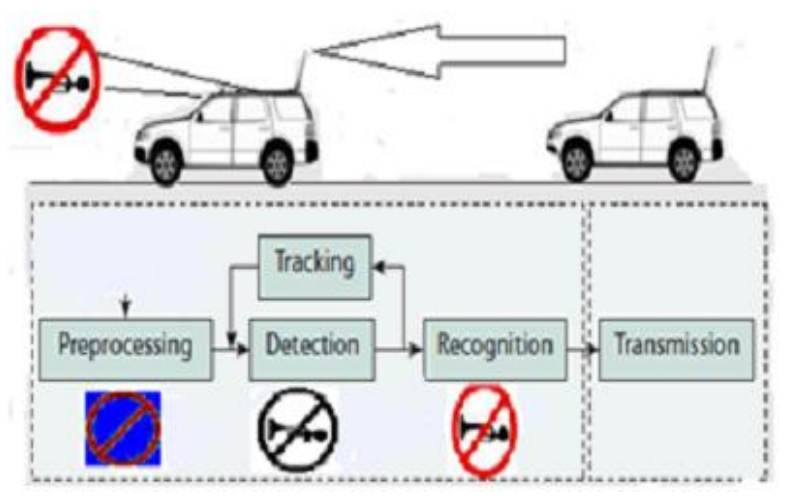

Figure 2: Traffic signs preprocessing, detection, recognition and transmission stages

During preprocessing, the image quality is enhanced so that it can be efficiently delivered to the subsequent stages. In the detection stage, the image is searched for different traffic signs and hence regions of interest (ROI) are calculated by using color and shape features of the sign. These ROI's are then analyzed in the recognition stage. After the signs are 
correctly recognized, they are then transmitted from one vehicle to another wirelessly.

In this paper, we have done a comparative study of all the methods of detection and recognition algorithms used in designing traffic sign recognition system. We have addressed all the issues and challenges in designing the traffic signs detection system. Table 1 discusses the various techniques used by various traffic sign recognition (TSR) system from preprocessing to recognition stage.

Table 1: Techniques used by various TSR system

\begin{tabular}{|c|c|c|}
\hline Stages & & Methods \\
\hline Preprocessing & \multicolumn{2}{|c|}{ Camera calibration and its resolution } \\
\hline \multirow{6}{*}{ Detection } & \multirow[t]{2}{*}{$\begin{array}{l}\text { Color } \\
\text { based }\end{array}$} & Color Thresholding \\
\hline & & Color Learning \\
\hline & \multirow{4}{*}{$\begin{array}{l}\text { Shape } \\
\text { Based }\end{array}$} & Edge Detection \\
\hline & & Template Algorithm \\
\hline & & $\begin{array}{l}\text { Machine learning } \\
\text { techniques }\end{array}$ \\
\hline & & Hough Transform \\
\hline \multirow[t]{2}{*}{ Recognition } & \multicolumn{2}{|c|}{ Template Matching } \\
\hline & \multicolumn{2}{|c|}{ Machine Learning } \\
\hline
\end{tabular}

\section{ISSUES AND CHALLENGES}

The various methods for which traffic signs detection must take into account the following:

Type of input- Video or Static images.

Weather conditions-Are the images taken in daylight or at night or in adverse conditions like rain, fog or snow.

Type of sensor- High or low resolution camera, grayscale or color sensor.

Processing method- Real time image capture or offline.

There are many issues that must be considered while designing any traffic signs recognition system. They are acquisition vision system, conditions of road, surrounding conditions, traffic sign state and size of image.

\subsection{Acquisition System}

The fundamental function of acquisition system is to capture the image or video so that it can be further processed. In fact, the camera should be properly calibrated and its dynamic range should be adjusted before capturing any scene. Recently, high dynamic range cameras (HDR) based on CMOS technology has begun to develop in field of driver assistance systems since they provide high contrast images during night. The position and resolution of cameras acquiring the images of signs are the main concern. If the cameras are placed too far away, then the image captured will be blurred and cannot be detected accurately. But if the camera is placed too close (maybe on bumper of car); it will capture images which can be affected by the climatic conditions. Hence the distance of camera from the image determines the quality of image. The traffic sign detected in the image is small and noisy if captured with a low resolution camera and the image would not be detected efficiently. Hence many researchers have tried to use cameras of resolution $640 * 480$ pixels. But practically this resolution is insufficient while dealing with signs which are far away from the camera. There can also be excessive tilt in camera plane while capturing the image. All of these factors can make it impossible to recover the useful information from the images.

\subsection{Surrounding Conditions}

The detected color of the image of the roadside sign can be different from the actual color due to illumination effects. The shape of image is also affected due to occlusion and weather conditions such as rain, snow or fog. Surrounding objects like buildings or trees may directly or indirectly affect the detection process.

\subsection{Traffic Sign State}

The traffic sign on the roadside may be damaged due to physical conditions of environment. This degradation of sign can be due to ultra violet radiations or due to inappropriate material used in making sign. There could be mechanical deformations like folding of edges. These could affect the shape and color of sign and can make detection process tedious.

\subsection{Size of Image}

The size of image appearing in the scene has an impact on the accuracy of detection and recognition process. If the signs are too small then they would not be detected as picking up of color and shape would be challenging task even for best computer vision algorithms. A large size image could provide enough and good quality information but requires a very large processing time. Hence a system should be designed so that it keeps track of signs from the point it become visible until a reasonable size that would be easily detected.

\section{TRAFFIC SIGN STAGES \\ 3.1 Preprocessing Techniques}

Preprocessing stage is essential for making the detection process faster. It not only considers the camera calibration but also enhance the quality of image. High dynamic range (HDR) cameras should be tested for sign detection for good quality images. Cmos Video Cameras should be used with high HDR. The authors in [1] proposed the techniques for both reducing the HDR and temporal effects. The image captured from the camera is of RGB nature. These images are very sensitive to lighting variations. To reduce the effect of lightning a proper color space conversion has to be done. The most important color spaces used for detection purpose are HIS, $\mathrm{YC}_{\mathrm{b}} \mathrm{C}_{\mathrm{r}}, \mathrm{HSV}, \mathrm{YUV}$ and RGB. The YUV color space give good results for red/yellow/orange signs, but it fails for white/black or low resolution colors. The authors in [2] used the ratios between the intensity of a given channel and the sum of all RGB channel intensities. The HSV (HueSaturation-Value) color space has been used in many works presented by Waite and Oruklu in [3] and authors in [4, 5]. This color space is preferred as it is ineffective to illumination effects. The interconversion formulas of RGB-HSV are nonlinear and hence the computational cost is very high. Moreover hue component changes with distance, dust and age. The $\mathrm{YC}_{\mathrm{b}} \mathrm{C}_{\mathrm{r}}$ color model has been used by authors in [6]. The chrominance component $(\mathrm{Y})$ was used to detect the signs. They worked on grayscale images as it presented good results regardless of lighting conditions (day/night/rainy/wet). Fang, Chen and Fuh in [7] used the concept of pre-stored hues to classify colors The reason was to store the hue values beforehand and the color label was calculated by calculating 
the similarity with all available hues, so that the classification that is most similar is chosen. Hence depending on the application the color space conversion can be chosen.

\subsection{Detection Techniques}

The detection process sends ROI's from the captured image to the recognition stage suppressing the background regions. There are many methods for correct detection of signs through color, shape and by using many machine learning techniques.

\subsubsection{Detection based on color}

Color segmentation involves either the use of any particular color space or any of the color learning methods.

\subsubsection{Techniques based on color thresholding}

Color based detection techniques aim to search the area of interest based on colors of interest using color based thresholding or segmentation techniques. Different color spaces can be used to detect the color of interest and separate it from background. Several models have been designed. In mid -1990s, several researchers used Hue-Saturation-Intensity (HIS) color space model. The authors in [8] used color thresholding to segment the image. They implemented the thresholding using a 16-bit look-up-table.

C.H Lai and C.C.Yu in [9] performed the color detection in the HSV color space. They concluded that the color saturation shows significant change when the videos are taken from different devices. Some authors [10] studied the effect of light on the color of traffic signs during day and night and concluded that the color of roadside image could get distorted due to light and this may affect the quality of images. However, they emphasized that outdoor illumination does not affect the RGB component differences for traffic signs. The authors in [11] emphasized on color segmentation as detection of shape is unreliable in urban areas. Unfortunately, all the segmentation algorithms based on color thresholding have to adjust their thresholds again and again. Hence color segmentation always requires a fixation of threshold.

\subsubsection{Techniques based on color learning methods}

Various learning methods have been implemented for traffic signs. Aoyagi and Asakura in [12] were the first ones, who worked in area of genetic algorithm (GA) for the sign detection. They detected the speed limit signs. They used smoothing filter and Laplacian filter to remove noise and then used genetic algorithm for sign detection. An algorithm based on Support Vector Machines (SVM) was presented by authors in [13] to classify pixels using color information.

The authors concluded that the segmentation based on learning algorithms give better results as compared to color thresholding segmentation. In [14], the detection is carried out using Haar wavelets obtained from Ada Boost algorithm. The authors have used the wavelet feature to solve many practical problems in real time.

\subsubsection{Detection based on shape}

Color based detection techniques are only useful if we are using high resolution CMOS color camera. Color based detection would not be effective during night when all the artificial lights are present which as confirmed by authors in [15]. Detection can be done by shape also. Shape detection is effective only when light illumination changes frequently. Moreover shape based detection works on grayscale images and this may help to meet global cost requirements. The shape is usually extracted either by their edges, gradient features, template matching or by Hough Transform.

\subsubsection{Shape detection using edges}

Most of the edge detection techniques depend on the contour or edge information of signs. The authors in [16] explained the detection of signs by using rectangular pattern matching algorithm. The areas detected as signs were defined under one scan window. The ellipse equation was also used to detect the circle in an image [17]. The authors in [18] explained the detection of rectangular and circular images of traffic sign using rectangular and circular detection algorithm. However, shape detection using edge features can only give accurate results in daytime only.

\subsubsection{Shape detection using template matching}

Shape can also be detected using template matching techniques. The captured image is matched with the images of known good traffic signs in the database. The matching is done by calculating the Hausdroff distance between the image captured and each template as implemented by authors in [19]. However, this technique is not suitable for real time applications as it requires many computations between template and ROIs for sign detection. Sheldon Waite and Oruklu in [20] applied this technique in face recognition and object matching. The main advantage of using template approach is that it can be modified to detect any objects.

\subsubsection{Detection based on machine learning techniques}

Machine learning algorithms like support vector machines (SVMs) and neural networks (NNs) can be used to extract shapes. SVMs and NNs are used for signs detection because of their ability to detect shapes accurately. Neural networks are trained for each set of signs. However, addition of more signs implies again training of network and hence manual selection of training samples. SVM's are invariant to rotation, translation and partial occlusions of road signs. MSERs (maximally stable extremal regions) work on the traffic symbols with white background. Although MSERs are a robust form of sign detection in complex scenes but they are computationally expensive. The authors in [21] explained the detection process by calculating the threshold at different levels ranging from 70 to 190 and their MSERs.

\subsubsection{Shape detection based on hough transform}

Hough transform is used to detect circles and lines based on curve fitting algorithm. The advantage of using Hough transform is that it is not sensitive to imperfect data and noise and also manages to detect occluded images. The authors in [22] used circular Hough transform for detection of European speed limit signs. However, the authors used rectangular edge detection based algorithm for detecting U.S speed limit signs.

\subsection{Recognition Techniques}

The recognition stage receives the ROIs from the detection stage that possibly contains one or more signs. Since there is huge number of traffic signs and moreover there can be distortions and occlusions on the signs, the recognition system should always be reliable. Traffic signs can be recognized by mainly two main approaches: template matching or machine learning techniques. 


\subsubsection{Recognition based on template matching}

This algorithm stores some sample sign images in database. Template matching was used by authors in [23] for classification. They calculated the distance between candidate regions came from detection stage and different sizes of template images in database. The template having minimum distance is the matched sign. However, template matching algorithm fails for tilted, rotated and partially hidden traffic signs.

\subsubsection{Recognition based on machine learning techniques}

There are many machine learning techniques like SVMs, Neural networks which can be used to recognize signs. The authors in [21] used the cascaded structure of support vectors classifiers and HOG features to recognize the signs. SURF (Speeded up Robust Feature) algorithm was used to match two traffic signs even if they are slightly rotated. The authors in [11] created a SURF database of various traffic signs templates. However, a fast internet connection is needed for better performance of tool. A mobile based application" Mansalakuna" was developed by authors in [23] that would detect signs through mobile phones. They depend on Global positioning system (GPS) and digital road maps. But again a fast internet connection is needed for application based traffic sign detection and recognition system.

\section{HARDWARE PERSPECTIVE OF TRAFFIC SIGNS IMPLEMENTATION}

While considering the hardware aspects, some algorithms have to be modified to overcome the real time constraint. To implement the TSR system to hardware, researchers are using Simulink graphical tool for modeling, simulation which is suitable for making image processing block. The authors in [24] had proposed FPGA based hardware implementation of road signs. The authors have used Matlab/Xilinx system Generator as it has a big advantage in terms of the conception time. This tool provide the user some special Xilinx building blocks to create the optimized designs for Xilinx FPGA's. Moreover, XSG operate on fixed point data which represent the wide range of formats for hardware implementation.

\section{CONCLUSION AND FUTURE SCOPE}

This research has divided the TSR system in three main stages as preprocessing; detection and recoginition.It describe each stages showing the various methods of detection and recognition and some techniques that can be used effectively for its hardware implementation. It was shown by some of the authors that the hardware implementation of TSR system can be optimized by using XSG tool. The use of this tool has a benefit in terms of conception time and hence can be embedded in any car equipped with high resolution cameras and GPS receiver. Table 2 illustrates the various sign detection and recognition methods used by researchers from the last 15 years.

\section{REFERENCES}

[1] Stefano Marsi, Gaetano Impoco, Anna Ukovich, Sergio Carrato and Giovanni Ramponi," Video Enhancement and Dynamic Range Control of HDR Sequences for Automotive Applications", EURASIP Journal on Advances in Signal Processing, Vol.2007 Article ID 80971.

[2] Arturo Escalera, Lius Moreno, Miguel Salichs and Jose Armingol,"Road traffic Sign Detection and
Classification", IEEE Transactions on Industrial Electronics, Vol.44, No.6, pp.848-859, December 1997.

[3] Sheldon Waite and Erdal Oruklu," FPGA-Based Traffic Sign Recognition for Advanced Driver Assistance Systems", Journal of Transportation Technologies, Vol.3, pp.1-16, November 2012.

[4] Chokri Souani, Hassene Faiedh and Kamel Besbes ," Efficient algorithm for automatic road sign recognition and its hardware implementation", Journal of Real Time Image Processing, Vol.9, pp.79-93,April 2013.

[5] Karla Brkic,"An overview of traffic sign detection methods", Department of Electronics, Microelectronics, Computer and Intelligent Systems Faculty of Electrical Engineering and Computing Unska, 2010.

[6] Ching-Hao Lai and Chia-Chen Yu, "An efficient realtime traffic sign recognition system for intelligent vehicles with smart phones", In Technologies and Applications of Artificial Intelligence (TAAI), 2010 IEEE International Conference , pp. 195-202, 2010.

[7] C.Y.Fang, S.W Chen and C.S. Fuh," Road sign detection and tracking", IEEE Transactions on Vehicular Technology, Vol. 52, No. 5, pp. 13291341.September 2003.

[8] Arturo Escalera, Luis E. Moreno, Miguel Angel Salichs and José Maria Armingol " Road traffic sign detection and classification",IEEE transactions on Industrial Electronics , Vol. 44, No. 6, pp. 848-859,December 1997.

[9] Ching- Hao Lai, and Yu Chia-Chen "An efficient realtime traffic sign recognition system for intelligent vehicles with smart phones", In IEEE International Conference on Technologies and Applications of Artificial Intelligence (TAAI), pp. 195-202, 2010.

[10] M. Benallal and J. Meunier,"Real-time color segmentation of road signs", IEEE Canadian Conference on Electrical and Computer Engineering, Vol.3, pp.1823-1826,2003.

[11] E. Oruklu, D. Pesty, J. Neveux and J. E .Guebey," Real Time Traffic Sign Detection and Recognition for in car Driver Assistance Systems", IEEE International Symposium on Circuits and System, pp.976-979,2012.

[12] Y. Aoyagi and T. Asakura " A study on traffic sign recognition in scene image using genetic algorithm and neural networks", IEEE International Conference on Industrial Electronics, Control and Instrumentation ,Vol. 3, pp. 1838-1843,1996.

[13] H.G.Moreno ， S.M.Bascón ， P.G.Jiménez.and S.F.Arroyo," Goal Evaluation of Segmentation Algorithms for Traffic Sign Recognition", IEEE Transaction on Intelligent Transportation Systems, Vol. 11, No. 4, pp.917- 930,2010.

[14] Claus Bahlmann, Ying Zhu, Ramesh Visvanathan, M. Pellkofer and K.Thorsten," A system for Traffic Sign detection ,tracking and recognition Using color, shape and motion Information", In IEEE proceedings of Intelligent Vehicles Symposium, pp. 255-260,2005.

15] Miguel Angel García-Garrido, Miguel Angel Sotelo and Ernesto Martin- Gorostiza," Fast traffic sign detection and recognition under changing lighting conditions", In IEEE Intelligent Transportation Systems Conference, pp. 811-816, 2006. 
[16] Anh-Tuan Hoang, Tetsush Koide and Masaharu Yamamoto," Low Cost Hardware Implementation for Traffic Sign Detection system", IEEE Asia Pacific Conference on Circuit and System, pp.363-366, 2014.

[17] Aurore Arlicot,Bahman Soheilian and Nicolas Paparoditis," Circular road sign extraction from street level images using color, shape and texture databases maps", Titled in International Archives of Photogrammetry, Remote Sensing and Spatial Informational services in IAPRS,Vol.38, pp. 205-210, 2009.

[18] Fabien Moutarde, Alexandre Bargeton, Anne Herbin and Lowik Chanussot," Robust on-vehicle real-time visual detection of American and European speed limit signs with a modular Traffic Signs Recognition system", In IEEE Intelligent Vehicles Symposium, Istanbul, Turkey, pp.1122- 1126,June 2007.

[19] De La Escalera, J. Ma Armingol and Mario Mata, Traffic Sign Recognition and Analysis for intelligent vehicles", Journal of image and vision computing, Vol.21, No.3, pp.247-258, 2003.
[20] S.Waite and E. Oruklu," FPGA-Based Traffic Sign Recognition for Advanced Driver Assistance Systems", Journal of Transportation Technologies, Vol.3, pp.116,2013

[21] J.Greenhalgh and M. Mehdi," Real-Time Detection and recognition of road traffic signs", IEEE Transactions on intelligent transportation systems, Vol.13, No.4, pp.1498-1506, 2012

[22] Par Karem and Oguz Tosun," Real-time traffic sign recognition with map fusion on multicore/many core architectures", In Acta Polytechnica Hungarica, Vol.9, No.2, pp.231-250, 2012.

[23] A. Karunalithika,R.P.Jayasundra,M.A.Rasamjan ,D.N. Senayanke and V.N.Vithana," Road sign identification application using image processing and augmented reality", International journal of advanced computer technology ,Vol. 4,Issue.11.pp.79-93,2015.

[24] Rihab Hamida, Abdessalam Abdelali and Abdellatif Mtibaa," Hardware implementation and validation of a traffic road sign detection and identification system", Journal of Real Time Image processing, pp. 1-18, 2016

\section{APPENDIX}

Table 2: Summary of various detection and recognition methods

\begin{tabular}{|c|c|c|c|}
\hline Authors & Year & Detection Algorithm & Recognition Algorithm \\
\hline Eclasera and Salichs [2] & 1997 & Color thresholding & Neural network \\
\hline $\begin{array}{c}\text { Benallal and Meunier } \\
\qquad[10]\end{array}$ & 2003 & Color segmentation & - \\
\hline Escelera [19] & 2003 & Genetic algorithm & Neural network \\
\hline Claus Bahlaam [14] & 2006 & Ada Boost algorithm. & Bayesian generative modeling. \\
\hline Mourtarde [18] & 2007 & $\begin{array}{l}\text { Shape detection through } \\
\text { Hough transform }\end{array}$ & Neural network \\
\hline Arlicot [17] & 2009 & color segmentation & SVM \\
\hline Oruklu [11] & 2013 & Color segmentation. & Template matching and Neural network \\
\hline Chokri Souani [4] & 2013 & Color segmentation & Neural network \\
\hline A.T .Hoang [16] & 2014 & $\begin{array}{l}\text { Shape detection through } \\
\text { rectangle matching algorithm }\end{array}$ & - \\
\hline Karunalithika[23] & 2015 & $\begin{array}{l}\text { OpenCV Library is used for } \\
\text { detection }\end{array}$ & Mobile based application \\
\hline Rihab Hamida [24] & 2016 & $\begin{array}{l}\text { Color segmentation based on } \\
\text { Xilinx System Generator }\end{array}$ & $\begin{array}{c}\text { Simulink model based on Xilinx System } \\
\text { Generator }\end{array}$ \\
\hline
\end{tabular}

